\title{
Criteria Catalogue for Collaborative Environments
}

\author{
Franca A Rupprecht \\ TU Kaiserslautern \\ Germany \\ rupprecht@cs.uni-kl.de
}

\author{
Taimur K Khan \\ TU Kaiserslautern \\ Germany \\ tkhan@cs.uni-kl.de
}

\author{
Gerrit van der Veer \\ Vrije Universteit Amsterdam \\ Netherlands \\ gerrit@acm.org
}

\author{
Achim Ebert \\ TU Kaiserslautern \\ Germany \\ ebert@cs.uni-kl.de
}

\begin{abstract}
Computer supported collaborative work supports the interaction and joint task solving between humans by setting a machine or computer in-between. Similarly, collaborative environments support decisionmakings incorporating different points of view and a variety of competences. As such, the identification and selection of the underlying requirements involved in collaboration is a challenging task. In order to facilitate this task, we performed a thorough literature review with the aim to discern such requirements. The result is a set of qualitative criteria that can be applied to generic computer supported collaboration environments.
\end{abstract}

Furthermore, the mapping of the generic catalogue to a specific collaboration task is demonstrated.

Teamwork support, Collaboration Software, Criteria, Computer supported Collaborative Work, $\mathrm{HCl}$

\section{INTRODUCTION}

Collaboration among stakeholders is an increasingly used practice for design, evaluation and concept balancing. This practice enables decision-making processes with a rich environment allowing different points of view and a variety of competencies. Reflecting and integrating all ideas and expertise is crucially important. Commonly, software tools are used to support this collaborative working style. According to Mittleman et al. (2008), most of these collaboration tools are developed for the mass market and do not suffice the specific needs of a collaborating group. Hence, these needs must be satisfied by the use of customized tools. The works' objective is to establish and provide a set of qualitative criteria that apply to generic collaboration environments that can substantially and holistically advance the productivity of collaborations. The specific adaption and utilization of the criteria catalogue will be demonstrated in a case study based on a task model of collaborative authoring.

\section{RELATED WORK}

An extensive literature review was performed covering the fields of Human Computer Interaction $(\mathrm{HCl})$, Computer-supported collaborative work (CSCW), cognitive science and social science. From this wide range of collected publications, we identified criteria for collaborative work dedicated to specific technology (Borghoff and Schlichter (2000)) like table tops (Scott et al. (2003)) or mobile devices (Donker and Blumberg (2011)). We extracted criteria of collaboration support systems in information visualization (Tobiasz et al. (2009)), visual analytics (Bren-nan et al. (2006)), business processes (Mundbrod (2012)), virtual reality (Liu et al. (2012)) and de-sign and engineering (French et al. (2014), Johnson et al. (2011), Briggs et al. (2010) and Briggs et al. (2003)). Also, existing work about single aspects of successful cooperation like awareness indication (BeaudouinLafon and Karsenty (1992), Dourish and Bellotti (1992)), as well as satisfaction and team effectiveness as investigated by (French and Kot-tke (2013), Jose' et al. (2014) and Rousseau and Aube' (2010)) have been considered. The criteria catalogue presented here provides an overview of the most important support features for collaboration systems and contains design recommendations to achieve the desired facilitation support. Explanations about the selection process is provided.

\section{COLLABORATIVE WORK}

Collaboration can be defined as a joint activity in which individual participants share an obligation to coordinate in individual autonomy in the service 
of progress towards a common goal (Johnson et al. (2011)). This definition indicates that different working styles and different work phases exist in collaborative work. Working styles can be classified into two groups: task performance (jointly working on a task) and single task performance (individual working on a task), where actors work individually by taking on responsibilities for directing their task accomplishment toward the achievement of the established team goals. In regards to computer support of team work additional attributes like the participants' location and the synchronicity of work performance needs to be considered. Therefore, the task performance can be identified upon the attributes location; indicating the physical location of the participants and division of labour, which describes that tasks are performed individually but the integration and collection of the results need to meet the requirements of all participants. Thus, we can distinguish among four types of working styles between which actors switch most likely during:

co-located cooperation;

distributed cooperation;

co-located single task performance;

distributed single task performance.

Figure1 depicts the underlying ontology, which refers to the main task phases in collaborative work sessions and serves as baseline to define collaborative task performance. Every collaboration

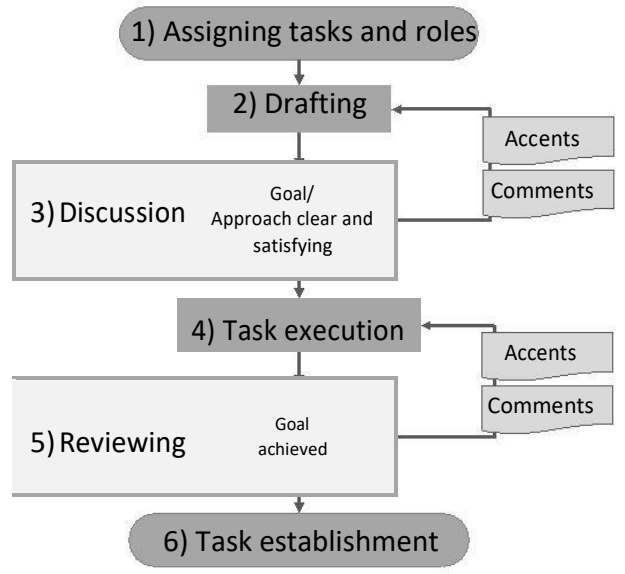

Figure 1: Conceptual framework of collaborative work

session starts with the assignment of tasks and roles to each actor that is facilitated by a software tool or performed in a group meeting setup beforehand (step 1). No active participation of actors lowers the motivation of the entire group and the quality of the overall results. In the next step, users (jointly or individually) create drafts of the desired goals and the approach for reaching these goals (step 2). This step is accompanied by continuous comments and feedback loops, which lead to group discussions (step 3). The outcome is a draft in which ideas and expertise from all participants are considered and integrated. Afterwards the working style changes to single task performance where the actual execution of the assigned tasks is performed (step 4). The phase of task execution is closely linked with the reviewing and revision phase (step 5) in which all ideas, comments and suggestion will be discussed and incorporated. The decision about accepting the output is the outcome of step 5 and leads to task establishment (step 6).

\section{CRITERIA IDENTIFICATION}

To identify all-embracing criteria of collaboration support systems, we observed existing theoretical and practical literature as mentioned in the related work and identified criteria, intended to support collaborative work. In the first step, the criteria have been collected, sorted and grouped in conformity with the proposed instructions (e.g. highlighting, screen sharing, etc.). Then, analogous criteria have been detected and integrated into single criterion definitions while additional criteria are deduced from existing ones. Additionally, the results of this classification were validated through extensive interviews and questionnaires, and by monitoring and observing collaborative design sessions. Afterwards a categorization based on the impact of the instructions has been performed (e.g. instruction: accentuating leads to impact: information sharing). The resulting catalogue of collaboration support criteria is presented below:

1. Content support refers to the active interaction and integration of the content by the actors, which is highly reliant on the underlying task. Drafting and task execution phases as depicted in Figure 1 are strongly related to the underlying task model and can be for example, a writing process, designing or creation. These criteria are required in adapted form, which are derived from the task models.

a. Content integration: Add, Associate, Modify, Delete.

b. Move: Change structures and appearances.

c. Judge: Render an opinion to the made contribution.

2. Information sharing involves functionalities and technical modules, which are used to share information in order to establish the knowledge basis for all actors.

a. Quickly retrieve context-relevant information: Ability to detect changes.

b. Access to shared objects: Ability to access and edit shared artefacts

c. Accentuating: Pointing, marking, annotate.

d. Track others approach: Can improve coordina-tion and skill transferability. 
e. Screen sharing: Ability to accentuate own or draw attention to others viewpoint.

f. Individual and/or shared workspaces: Allows performing single tasks and keep track of overall goal.

3. Coordination support refers to the spectrum of tasks that are used to support the coordination of work packages and the coordination between actors.

a. Jurisdiction: Assignment of tasks, roles, responsibilities, rights.

b. Transformations: Transitions between personal and group work, between activities, and between tool and external work.

c. Alert mechanisms: Notification of changes or of required user input.

d. Awareness Support: Amplifies coordination and communication.

e. Community Support: Online documentation and strength of the community.

f. Team structure and size: Ability to create team structures and optimal team sizes.

g. Changing work styles: Ability to change between single and group work.

4. Communication support describes the support of advanced and unimpeded communication among actors to bridge spatial gaps [2].

a. Communication in group and or individual: For public and private conversations.

b. Discussion tool: Rich and powerful communication channels.

c. Encrypted Communication: Increases actors reliance in the technology.

5. Compliance support relates to rules or guidelines that should be fulfilled to conduct a well thought out decision making process.

a. Avoid team debates: If actor has to explain himself with low accordance the individuals satisfaction is reduced.

b. Group process training: Supports the sense of cohesiveness and shared goal.

c. Reflecting all individuals' notions/opinions: Feeling of left out arises frustration.

d. Use guidelines and defined restrictions instead of strict rules: Guidelines for task performance, discussion, and decision making.

e. Involving all actors: Actors without a task or role are not part of the work team.

f. Team self-managing behaviours: The ability of actors to collaboratively assume responsibili-ties for directing their task accomplishment to-ward the achievement of the established team goals.

6. Content management refers to the action execution of dynamical content manipulation by actors and granting valid and reliable database entries. a. Action parameter: Synchronicity of action and identifiability of actors.

b. Access Control: Allocation of access rights.

c. Session Persistence: Degree to which contributions are ephemeral or permanent.

d. Consistency and interactivity: Causality, concurrency, simultaneity, instantaneity.

7. Usability involves next to user satisfaction to the degree of efficiency and effectiveness of the technology, including the acceptability by users.

a. Reliability: Same results are achievable with different actors.

b. Reusability: Session/ results/ configurations can be recorded and reused.

c. Transferability of skills: Degree of apprenticeship of a novel actor in order to gain insights and being able to conduct work practices on their own.

d. Flexible actor arrangements: Configurable collaborative components scaling over different types and sizes of input devices.

e. Guidance: Provision of facilitation support.

f. Generalizable and ease of maintenance: Generic for different projects/domains.

8. User experience describes the actors emotions and attitudes about using the technology.

a. Natural interpersonal interaction: Urges collaborative interaction/communication.

b. High user satisfaction and motivation: Amplifies active participation.

c. Intuitive and simple technology: The tool is easy to understand and easy to use.

d. Reduced cognitive load of actors: Actors should be able to focus on the task.

e. No all-embracing knowledge/expertise needed: actors only learn the techniques needed to conduct own domain related actions.

\section{AN EXAMPLE DOMAIN}

We demonstrate the use in practice of the described catalogue by illustrating the impacts of the interdisciplinary collaboration requirements using a dedicated task model of collaborative authoring as introduced by Kulyk et al. (2011).

\subsection{Collaborative Authoring}

The aim of collaborative authoring is to combine input of multiple actors in order to create a written document. Similar to the ontology of collaborative work in Figure 1, Figure 2 sketches the activities performed in collaborative authoring. The general steps are adopted to the task, for example "Task execution" is refined into the tasks of "Writing" and "Formatting". The collaborative session starts 
with high-level activities, like the conduction of a planning meeting to define the concept of the outcome. A document outline may be defined and writing tasks are distributed among the authors. The assignment of work can be done by a single individual or through mutual agreement of the authors. Subsequently, medium level activities like writing single chapters, creating graphs or collecting references, are commonly performed by individual actors in a single task setting. Low-level activities describe sub tasks, which are performed in order to accomplish medium level tasks: combining document fragments, note taking, etc. The performance of medium level activities is connected with continuous feedback loops provoked by commenting and accentuating.

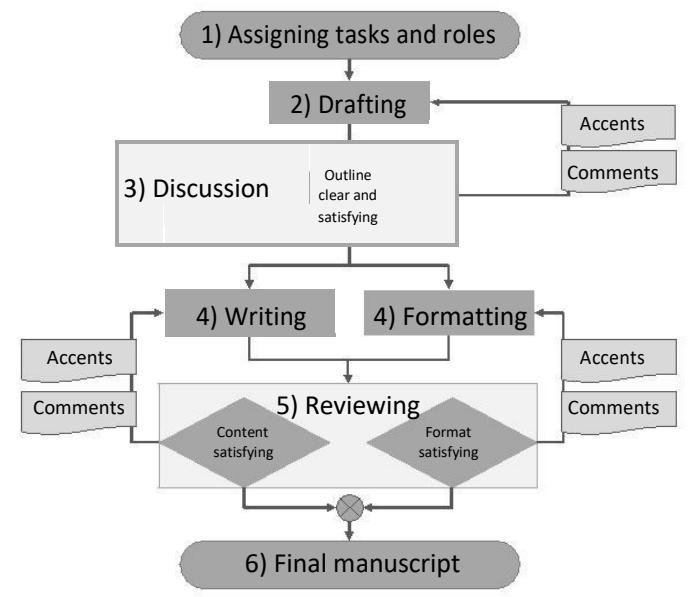

Figure 2: Activity model of collaborative authoring.

\subsection{Criteria Identification}

During the process of collaborative authoring, the following medium level activities are identified: writing, formatting, commenting, accentuating, reviewing and revising. These activities can be directly adopted as features that have to be supported by the system. By using the catalogue these features are refined by choosing the corresponding criteria. Finally, the selected criteria for supporting the task of collaborative authoring can be listed:

1. Content support

Content

integration Move

Judge

2. Information sharing Quickly retrieving context-relevant information Access and edit shared artifacts

Accentuating

Shared and individual workspaces

3. Coordination support Jurisdiction

Transformation: personal and group work; tool and external tool

Changing work styles
4. Communication support Group discussion tool

5. Compliance support Involving all actors

6. Content management Synchronization of actions Session persistence Consistency and interactivity

7. Usability

Re-usability of the results and configurations

8. User experience High user satisfaction and motivation Intuitive and simple technology

To highlight the selection process we discuss the criteria selection of Coordination support from the generic catalogue adopted to the example domain. Jurisdiction (3.a) is crucial to assign tasks and roles to guarantee that every actor can actively participate. Transitions between personal and group work (3.b) including the ability to change between working styles (3.g) is important to enable the authors to make own drafts individually and merge it with the group work when satisfied with the content. Also transitions between the collaborative authoring tool and external tools should be provided to utilize the resulting document.

Excluded from the general list have been the criteria 3.c, 3.d, 3.e and 3.f. Alert mechanism (3.c) are used to notify authors about when and where did changes occur. Awareness support (3.d) enables to recognize who is currently working on the document. These criteria help to coordinate the process and probably lead to a higher user satisfaction, but they are not crucial to successfully perform the overall task, as tested in the collaborative authoring tool Overleaf Limited (2016). Community support (3.e) of the document preparation system (LaTex vs. WYSIWYG word processors like Microsoft Word), might be necessary but it is not considered as requirement of the collaboration environment. As the team structure in the collaborative authoring process might not be multi-layered, it is not crucial to create additional hierarchies in the team structure (3.f). Hence, these criteria are not selected in this case.

\section{CONCLUSION AND FUTURE WORK}

In this paper we presented a generic criteria catalog for collaborative environments and demonstrated it's use in practice on the task model of collaborative authoring. The presented criteria catalog will be used further on to identify software architecture modules of new systems and can serve as a guideline for evaluating existing collaboration systems.

\section{ACKNOWLEDGEMENTS}

This research was funded by the German Research Foundation (DFG) as part of the IRTG 2057. 


\section{REFERENCES}

Beaudouin-Lafon, M. and A. Karsenty (1992). Transparency and awareness in a real-time groupware system. In Proceedings of the 5th annual ACM symposium on User interface software and technology, pp. 171-180. ACM.

Borghoff, U. M. and J. H. Schlichter (2000). Computer-supported cooperative work. In Computer-Supported Cooperative Work, pp. 87141. Springer.

Brennan, S. E., K. Mueller, G. Zelinsky, I. Ramakrishnan, D. S. Warren, and A. Kaufman (2006). Toward a multi-analyst, collaborative framework for visual analytics. In Visual Analytics Science And Technology, 2006 IEEE Symposium On, pp. 129136. IEEE.

Briggs, R. O., G.-J. De Vreede, and J. F. Nunamaker Jr (2003). Collaboration engineering with thinklets to pursue sustained success with group support systems. Journal of Management Information Systems 19(4), 31-64.

Briggs, R. O., G. L. Kolfschoten, G.-J. De Vreede, C. C. Albrecht, and S. G. Lukosch (2010). Facilita-tor in a box: Computer assisted collaboration engineering and process support systems for rapid development of collaborative applications for highvalue tasks. In System Sciences (HICSS), 2010 43rd Hawaii International Conference on, pp. 1- 10. IEEE.

Donker, H. and M. Blumberg (2011). Computer support of team work on mobile devices. In International Conference on Human-Computer Interaction, pp. 38-47. Springer.

Dourish, P. and V. Bellotti (1992). Awareness and coordination in shared workspaces. In Proceedings of the 1992 ACM conference on Computersupported cooperative work, pp. 107-114. ACM.

French, D. J., B. Stone, T. T. Nysetvold, A. Hepworth, and W. E. Red (2014). Collaborative design principles from minecraft with applications to multiuser cad. In ASME 2014 International Design Engineering Technical Conferences and Computers and Information in Engineering Conference. American Society of Mechanical Engineers.

French, K. A. and J. L. Kottke (2013). Teamwork satisfaction: Exploring the multilevel interaction of teamwork interest and group extraversion. Active Learning in Higher Education.
Johnson, M., J. M. Bradshaw, P. J. Feltovich, R. R. Hoffman, C. Jonker, B. van Riemsdijk, and M. Sierhuis (2011). Beyond cooperative robotics: The central role of interdependence in coactive design. IEEE Intelligent Systems 26(3), 81-88.

Jose,' M., V. Ferron'-V'ılchez, and N. Ortiz-de Mandojana (2014). Team decision making and individual satisfaction with the team. Small Group Research 45(2), 198-216.

Kulyk, O., D. Vyas, O. Kubbe, A. Ebert, et al. (2011). Task modeling for collaborative authoring. In Proceedings of the 29th Annual European Conference on Cognitive Ergonomics, pp. 171178. ACM.

Limited, W. (2016). overleaf for authors - write and collaborate online with latex.

Liu, H., M. Bowman, and F. Chang (2012). Survey of state melding in virtual worlds. ACM Computing Surveys (CSUR) 44(4), 21.

Mittleman, D. D., R. O. Briggs, J. Murphy, and A. Davis (2008). Toward a taxonomy of groupware technologies. In Groupware: Design, Implementation, and Use, pp. 305-317. Springer.

Mundbrod, N. (2012). Business Process Support for Collaborative Knowledge Workers. Ph. D. thesis, Faculty of Engineering and Computer Science Institute of Databases and Information Systems Master Thesis, Ulm University.

Rousseau, V. and C. Aube' (2010). Team selfmanaging behaviors and team effectiveness: The moderating effect of task routineness. Group \& Organization Management 35(6), 751-781.

Scott, S. D., K. D. Grant, and R. L. Mandryk (2003). System guidelines for co-located, collaborative work on a tabletop display. In ECSCW 2003, pp. 159178. Springer.

Tobiasz, M., P. Isenberg, and S. Carpendale (2009). Lark: Coordinating co-located collaboration with information visualization. Visualization and Computer Graphics, IEEE Transactions on 15(6), 1065-1072. 